Published in final edited form as:

Nat Genet. 2008 May ; 40(5): 529-537. doi:10.1038/ng.129.

\title{
Soluble epoxide hydrolase is a susceptibility factor for heart failure in a rat model of human disease
}

\author{
Jan Monti ${ }^{1,2,9}$, Judith Fischer ${ }^{1,9}$, Svetlana Paskas $^{1}$, Matthias Heinig ${ }^{1,3}$, Herbert Schulz ${ }^{1}$, \\ Claudia Gösele $^{1}$, Arnd Heuser ${ }^{1,2}$, Robert Fischer ${ }^{1,2}$, Cosima Schmidt ${ }^{1}$, Alexander \\ Schirdewan ${ }^{2}$, Volkmar Gross ${ }^{1}$, Oliver Hummel ${ }^{1}$, Henrike Maatz ${ }^{1}$, Giannino Patone ${ }^{1}$, Kathrin \\ Saar $^{1}$, Martin Vingron ${ }^{3}$, Steven M Weldon ${ }^{4}$, Klaus Lindpaintner ${ }^{5}$, Bruce D Hammock ${ }^{6}$, Klaus \\ Rohde $^{1}$, Rainer Dietz ${ }^{1,2}$, Stuart A Cook ${ }^{7}$, Wolf-Hagen Schunck ${ }^{1}$, Friedrich C Luft ${ }^{1,8}$, Norbert \\ Hubner $^{1}$
}

${ }^{1}$ Max-Delbrück Center for Molecular Medicine, Robert-Rössle-Strasse 10, 13125 Berlin, Germany. ${ }^{2}$ Department of Clinical and Molecular Cardiology, Franz-Volhard Clinic, HELIOS, Charité-Universitä tsmedizin Berlin, Schwanebecker Chaussee 50, 13125 Berlin, Germany. ${ }^{3}$ Department of Bioinformatics, Max-Planck-Institute for Molecular Genetics, Ihnestraf3e 63-73, 14195 Berlin, Germany. ${ }^{4}$ Boehringer Ingelheim Pharmaceuticals Inc., 900 Ridgebury Road, Ridgefield, Connecticut 06877-0368, USA. ${ }^{5}$ F. Hoffmann-La Roche, Grenzacherstrasse 124, 4070 Basel, Switzerland. ${ }^{6}$ Departments of Entomology and Nutrition and Cancer Research Center, University of California at Davis, One Shields Avenue, Davis, California 95616-8584, USA. ${ }^{7}$ National Heart and Lung Institute, Imperial College, Dovehouse Street, London, SW3 6LY, UK. ${ }^{8}$ Department of Nephrology/Hypertension, Franz-Volhard Clinic, HELIOS, Charité-Universitä tsmedizin Berlin, Schwanebecker Chaussee 50, 13125 Berlin, Germany. ${ }^{9}$ These authors contributed equally to this work.

\section{Abstract \\ We aimed to identify genetic variants associated with heart failure by using a rat model of the human disease. We performed invasive cardiac hemodynamic measurements in $\mathrm{F}_{2}$ crosses between spontaneously hypertensive heart failure (SHHF) rats and reference strains. We combined linkage analyses with genome-wide expression profiling and identified $E p h \times 2$ as a heart failure susceptibility gene in SHHF rats. Specifically, we found that cis variation at Ephx2 segregated with heart failure and with increased transcript expression, protein expression and enzyme activity, leading to a more rapid hydrolysis of cardioprotective epoxyeicosatrienoic acids. To confirm our results, we tested the role of Ephx2 in heart failure using knockout mice. Ephx2 gene ablation}

Correspondence should be addressed to N.H. (nhuebner@mdc-berlin.de). AUTHOR CONTRIBUTIONS

N.H. and J.M. developed the project. N.H. directed the project. J.F., S.P., C.G., C.S., H.M., G.P., K.S., S.A.C. and W.-H.S. performed genetic and biochemical analysis. J.M., A.H., R.F., A.S., V.G., R.D. and F.C.L. performed and analyzed physiological experiments. M.H., H.S., O.H., M.V. and K.R. carried out statistical analysis. B.D.H., S.M.W., K.L. and S.A.C. contributed materials. N.H. wrote the paper with J.M., J.F. and F.C.L.

URLs. SNPs used are available at http://www.ensembl.org and microsatellite markers at http://rgd.mcw.edu/.

Accession codes. Scanned microarray data have been submitted to Array-Express with accession code E-TABM-418. Ephx2 DNA variants have been submitted to dbSNP: ss86236892, ss86236893, ss86236894, ss86236895, ss86236896, ss86236897, ss86236898, ss86236899 and ss86236900. 
protected from pressure overload-induced heart failure and cardiac arrhythmias. We further demonstrated differential regulation of $E P H X 2$ in human heart failure, suggesting a cross-species role for Ephx2 in this complex disease.

Heart failure is an epidemiologically important disease, with $B 30 \%$ mortality at 1 year after diagnosis ${ }^{1-3}$. Most affected individuals have impaired systolic function with a reduced ejection fraction; their prognosis is particularly poor ${ }^{3}$. Heart failure is thought to result from complex interactions between genetic susceptibility and lifestyle and environmental factors ${ }^{4}$. Hypertension is one of the major risk factors ${ }^{5,6}$. Starting from high blood pressure, the pathophysiological cardiac remodeling cascade proceeds to left ventricular hypertrophy $(\mathrm{LVH})$ as a primarily adaptive process, which then proceeds to left ventricular dilatation, decreased systolic function and cardiac arrhythmias in some individuals, whereas others retain stable systolic function without clinical signs of heart failure. Elucidation of genetic factors has been limited by heterogeneity and by gene-environment interactions, as well as by the confounding presence of hypertension that serves as the driving force but is not sufficient as an intermediary phenotype. Familial predispositions contribute to heart failure in humans ${ }^{7}$. We used the spontaneously hypertensive heart failure (SHHF) rat, an inbred, genetically homogenous rat model that mirrors human hypertension-associated heart failure $^{8}$. SHHF rats not only develop heart failure late in life after high blood pressure and left ventricular hypertrophy have developed ${ }^{9}$, but also show many of the associated transcriptional and metabolic features of the human disease ${ }^{10-12}$. The model's genetic propensity is underscored by the fact that a closely related strain, the stroke-prone spontaneously hypertensive (SHRSP) rat, does not develop heart failure despite similarly elevated blood pressure.

We conducted a segregation analysis in $\mathrm{F}_{2}$ hybrids bred from SHHF and SHRSP, thus removing blood pressure and LVH as confounding variables. To test the overall blood pressure- and cardiac hypertrophy-related impact on the development of heart failure, we generated a second $\mathrm{F}_{2}$ cross-bred from SHHF and the normotensive Wistar-Kyoto (WKY) control rat. We integrated linkage analysis of hemodynamic phenotypes with cardiac genome-wide expression profiling in the $\mathrm{F}_{2}$ population, a strategy others and we have successfully used earlier to elucidate complex traits ${ }^{13-23}$. We identified the gene encoding soluble epoxide hydrolase $E p h x 2$, as a heart failure susceptibility gene. Finally, we validated our findings using Ephx2 knockout mice and expression studies in human heart failure.

\section{RESULTS}

\section{Determination of cardiac phenotypes}

We catheterized the left ventricle to precisely measure aortic and left ventricular enddiastolic pressures (LVEDP) and left ventricular end-diastolic volumes (LVEDV), as well as to construct pressure-volume loops. We found similar blood pressure and heart weight:body weight ratios, indicating LVH, in both SHHF and SHRSP compared to WKY rats. SHHF rats showed substantial left ventricular dilatation and reduced left ventricular ejection fraction, consistent with previous observations ${ }^{24}$ (Supplementary Fig. 1 online). Both SHHF 
and SHRSP rats had elevated LVEDP, with higher values in SHHF rats (Supplementary Fig. $1)$.

Blood pressure variance and LVH variance were similar or smaller in the $\mathrm{F}_{2}(\mathrm{SHHF} \times$ SHRSP) population than among progenitor strains, showing that blood pressure and LVH did not segregate in this cross. In contrast, the ejection fraction showed a large variance, ranging from $14 \%$ to $82 \%$ with a near normal distribution, consistent with the independent segregation of multiple alleles. In addition, we obtained quantitative hemodynamic parameters for LVEDP, LVEDV and left ventricular contractility (Dpressure/Dtime (mm $\left.\mathrm{Hg} / \mathrm{s}), \mathrm{d} P / \mathrm{d} t_{\max }\right)$ (Supplementary Table 1 online). In contrast to $\mathrm{F}_{2}(\mathrm{SHHF} \times \mathrm{SHRSP})$ rats, $\mathrm{F}_{2}(\mathrm{SHHF} \times \mathrm{WKY})$ rats showed decreased blood pressure and reduced LVH (Supplementary Table 1).

The $\mathrm{F}_{2}(\mathrm{SHHF} \times \mathrm{WKY})$ data show that the overall lower average blood pressure was paralleled, on average, by a higher ejection fraction, decreased LVEDP, lower LVEDV, improved contractility and decreased B-type natriuretic peptide (Supplementary Table 1). These observations support our contention that genetic factors contributing to the complex heart failure phenotype should be assessed in an experimental cross that equalizes the influences of high blood pressures and LVH as permissive heart failure factors, thus removing them as confounders. The $\mathrm{F}_{2}(\mathrm{SHHF} \times \mathrm{SHRSP})$ population that we characterized fulfills both requirements.

\section{Heart failure phenotypes map to rat chromosome 15}

We genotyped all $\mathrm{F}_{2}$ rats as described in the Supplementary Note online. In $\mathrm{F}_{2}(\mathrm{SHHF} \times$ SHRSP), a locus on rat chromosome 15 centered at marker D15Rat10 showed statistically highly significant linkage (lod score 4.3) to left ventricular ejection fraction, the principal clinical parameter used to characterize the failing heart (Fig. 1). At the same locus, we further demonstrated statistically significant linkage for cardiac contractility and highly suggestive linkage to LVEDV. The linkage peak on chromosome 15 for LVEDV, however, was some $20 \mathrm{cM}$ away (Fig. 1). Nonetheless, the SHHF allele was invariably associated with cardiac dysfunction. Grouping $\mathrm{F}_{2}$ rats according to zygosity at D15Rat10 did not show any genotype association with blood pressure or $\mathrm{LVH}$, as determined by analysis of variance (data not shown). This finding indicated that linkage to this locus was independent of blood pressure. Our data indicated that this quantitative trait locus (QTL) strongly affected heart failure in an additive mode, accounting for $15.1 \%$ of the phenotypic variance observed for the ejection fraction. As we observed clustering of three distinct clinical traits (ejection fraction, LVEDV and contractility) at D15Rat10 with varying degrees of significance, we speculated that a composite quantitative trait variable could extract further genetic information from this locus. Using principal component analysis, we next derived a composite phenotype for each $\mathrm{F}_{2}(\mathrm{SHHF} \times \mathrm{SHRSP})$ rat from three traits (ejection fraction, LVEDV and $\mathrm{d} P / \mathrm{d} t_{\max }$ ) and then subjected this composite trait to linkage analysis. This analysis resulted in a statistically more significant linkage at D15Rat10 (lod 4.8; Fig. 1), suggesting a complex but directional interplay of these phenotypes in the predisposition of heart failure at this locus, as well as indicating the limitations of individual, reductionist parameters as surrogate measurements for a complex phenotype in general. In contrast to the 
above, we found no evidence for linkage of ejection fraction in the $\mathrm{F}_{2}(\mathrm{SHHF} \times \mathrm{WKY})$ population anywhere in the genome (Supplementary Note and Supplementary Fig. 2 online).

\section{Transcript profiling in parental strains and $F_{2}$ populations}

Within the region of linkage to heart failure on chromosome 15, we identified only two transcripts that were differentially expressed with a false discovery rate (FDR) of $05 \%$ between the parental SHRSP and SHHF strains and showed a cis-regulated expression QTL(eQTL) at an FDR 05\% in the $\mathrm{F}_{2}(\mathrm{SHHF} \times \mathrm{SHRSP})$ population, consistent with the close relatedness of SHHF and SHRSP rats used in this experiment (Supplementary Note). Of the two cis-regulated transcripts, Ephx 2 showed the strongest genetic evidence for significant allele-specific regulation in the $\mathrm{F}_{2}(\mathrm{SHHF} \times \mathrm{SHRSP})$ population. The linkage peak (genome-wide corrected $P \bigcirc 10^{-6}$; FDR $\bigcirc 10^{-4}$; Supplementary Fig. 3 online) for the Ephx2 eQTL was at D15Rat10, the same marker that also defined the peak of the heart failure QTL on chromosome 15 in this cross. D15Rat10 is the nearest marker to Ephx2 and is located within $2.7 \mathrm{Mb}$, based on the rat genome reference sequence version 3.4. This finding suggested that Ephx2 transcript levels are regulated in cis at the Ephx2 gene itself and coincide with the peak of linkage to the heart failure QTL. The second cis-acting eQTL gene in this region was Mmp14. Even though it was located within the 95\% confidence interval encompassing the heart failure QTL, Mmp14 was located at a distance of more than $10 \mathrm{Mb}$ from the heart failure QTL peak marker D15Rat10. Strongest eQTL linkage of Mmp14 was observed with marker D15Rat83 (genome-wide corrected $P \bigcirc 10^{-3}$; FDR o $0.05)$. Based on statistical significance and proximity to the heart failure peak marker, we thus prioritized $E p h \times 2$ as candidate for further investigations.

\section{Sequence analysis of Ephx2}

We next aimed to elucidate regulatory sequence variations underlying the observed Ephx2 cis-regulated eQTL and to screen for coding sequence differences possibly altering protein function. Sequencing 5,000 bp upstream of the first exon, we found three SNPs and a 2-nt deletion in the putative Ephx2 promoter in SHHF as compared to SHRSP and WKY rats (Fig. 2). Genomic sequencing of the entire coding exon sequence, including adjacent splice sites, revealed two synonymous and three nonsynonymous SNPs (Fig. 2).

\section{Ephx2 is a heart failure susceptibility gene}

We established that Ephx2 was genetically linked to the peak marker of linkage for heart failure D15Rat10 by linkage analysis (Supplementary Note). The allelic status at Ephx2 accounted for $64 \%$ of the expression differences observed, with highest expression values associated to the SHHF genotype (Fig. 3a,b).

We measured Ephx2 abundance by protein blot analysis. Protein amounts in parental rats and $\mathrm{F}_{2}$ rats grouped according to zygosity at D15Rat10 mirrored the differences observed at the transcript level (Fig. 3c). Ephx2 encodes the soluble epoxide hydrolase (sEH), which hydrolyzes cardioprotective epoxyeicosatrienoic acids (EETs) ${ }^{25}$. To test the functional relevance - that is, effect on the substrate—of the allele-specific upregulation of Ephx2, we measured enzymatic hydrolase activity of Ephx2 in cardiac tissue of the parental and $\mathrm{F}_{2}$ rats grouped by zygosity at the peak marker D15Rat10. Our results demonstrated that the SHHF 
allele-specific upregulation of the transcript and protein also segregated with an augmented cardiac sEH activity (Fig. 3d). Finally, we directly measured the total amount of 14,15-EETs in the heart of parental and $\mathrm{F}_{2}$ rats, again grouped according to genotype. This analysis showed an effect of genotype on the total amount of cardioprotective EETs, the amount being significantly reduced when Ephx 2 was transcribed from the SHHF allele (Fig. 3e). Taken together, the results suggest that genetic variation in cis leads to upregulation of the $E p h \times 2$ transcript, elevated protein, increased hydrolysis of EETs and a decrease of cardioprotective EETs in the heart mediated by the Epxh2SHHF allele. The colocalization of this pathophysiological cascade triggered by the Ephx $2 \mathrm{eQTL}$ with the mapping of a heart failure QTL strongly suggests that Ephx2 contributes to the observed heart failure QTL.

This interpretation of the data is corroborated by the fact that, in all instances, the $E p h \times 2^{S H H F}$ allele was associated not only with upregulation of the Ephx 2 transcript and protein expression, but also with heart failure in the $\mathrm{F}_{2}$ population. Using the $10 \%$ upper and lower values of the tails of the ejection fraction distribution from $\mathrm{F}_{2}(\mathrm{SHHF} \times \mathrm{SHRSP})$ rats, we observed a marked correlation between $E p h \times 2$ transcript levels and ejection fraction (correlation coefficient $r^{2} 1 / 4-0.219, P \bigcirc 0.01$ ); rats with low ejection fraction had higher $E p h \times 2$ transcript levels, whereas rats with high ejection fraction had lower transcript levels. No correlation was detected when the entire dataset was analyzed $\left(t^{2} 1 / 4-0.048, P\right.$ not significant).

Ephx 2 promoter deletion creates an AP-1 binding site in SHHF To test whether the allelic promoter variants influence $E p h \times 2$ gene expression, we performed luciferase reporter assays and compared the variant promoters among SHHF, SHRSP and WKY rats. We found a strong increase in promoter activity with the SHHF compared to the SHRSP allele (Fig. 4a). The findings are consistent with the observed cis-regulated $\mathrm{eQTL}$ in $\mathrm{F}_{2}(\mathrm{SHHF} \times \mathrm{SHRSP})$, in which the SHHF allele was the allele that showed higher expression (Fig. 3a,b).

We also confirmed the effect of allele-specific regulatory variation using allele-specific realtime PCR of cDNA from heart tissue of ten $\mathrm{F}_{1}(\mathrm{SHHF} \times \mathrm{SHRSP})$ rats. This experiment confirmed that the expression of Ephx 2 was regulated in cis and that the $E p h \times 2^{S H H F}$ allelespecific transcript levels were significantly elevated compared to the Ephx $2^{S H R S P}$ allele $\left(P^{1 / 4}\right.$ $8 \times 10^{-12}$; Supplementary Fig. 4 online). Using computational predictions, we identified a consensus AP-1 (activator protein 1) transcription factor binding site in the Ephx2 promoter that exactly covered the 2-nt deletion in the SHHF strain (Fig. 4b). To investigate whether the mutated AP-1 binding site affected AP-1 binding in vitro, we performed electrophoretic mobility-shift assays; these showed specific AP-1 binding to the SHHF promoter, whereas AP-1 binding in SHRSP was abolished (Fig. 4b).

\section{SNPs in the coding region do not alter hydrolase activity}

Sequencing detected three nonsynonymous SNPs that could potentially affect Ephx2 enzymatic activity (Fig. 2). Conceivably, an allele-specific altered protein could confound the results attributed to allele-dependent expression differences. We demonstrated by in vitro expression of recombinant proteins that both SHHF and SHRSP Ephx2 proteins were equally active, which excludes a substantial effect of polymorphisms on the enzyme activity (Supplementary Fig. 5 online). Thus, the observed allele-dependent differences in the 
hydrolysis of 14,15-EET (Fig. 3d,e) were due to allele-specific expression differences of Ephx2.

\section{Ephx2 pathway shows genotype-dependent coregulation}

Because the transcriptional regulation of pathways related to $E p h \times 2$ function is of major interest, we next focused on transcripts of the arachidonic acid metabolic pathway that includes several important enzymatic reactions in the generation of epoxides (for example, 14,15-EETs) from arachidonic acid and the enzymes that regulate arachidonic acid synthesis or beta-oxidation, according to the Kyoto Encyclopedia of Genes and Genomes. We ranked the genes from the arachidonic acid metabolic pathway according to their association with the heart failure locus on chromosome 15 . None of these genes had a genome-wide significant eQTL at this locus when correcting for all transcripts tested. Nevertheless, we observe a concerted differential expression - in trans to Ephx2 - of the top-ranking genes, with individual $P$ values $\bigcirc 0.1$. Applying this relaxed filtering resulted in a set of genes containing six members of the $C Y P$ superfamily and three members of the PLA2 superfamily that will be referred to as the Ephx2 pathway (Fig. 5). To quantify the small but consistent effect, we computed a score for the association of gene expression at the level of the whole pathway with a genetic marker, which was applied as a genome-scan over all genetic markers used in $\mathrm{F}_{2}(\mathrm{SHHF} \times \mathrm{SHRSP})$. We found that the maximum association of the Eph $\times 2$-pathway transcripts occurred at the Ephx2 locus and that the pathway association score had genome-wide significance $\left(P^{1 / 4} 2.4 \times 10^{-5}\right)$.

\section{Ephx2 gene ablation protects from cardiac dysfunction}

To corroborate our findings that $E p h \times 2$ is involved in the pathogenesis of hypertensioninduced heart failure, we next studied $E p h \times 2$ knockout mice. We hypothesized that $E p h \times 2$ knockout mice could represent an extreme opposite compared to the detrimental effects associated with elevated Ephx 2 expression observed in $\mathrm{F}_{2}(\mathrm{SHHF} \times \mathrm{SHRSP})$.

At baseline, Ephx2 null mice and C57BL/6ByJ wild-type controls had normal blood pressure and normal left ventricular function (Fig. 6a,b) without signs of LVH. To investigate cardiac function under pressure overload conditions similar to those we observed in SHHF rats, we used prolonged ( 3 weeks) chronic intraperitoneal infusion of the potent vasoactive peptide angiotensin II (Ang II) at high dosages. Because the Ephx2 promoter harbors an Ang II-inducible AP-1 binding site that is also predicted in mouse, this experimental strategy was expected to amplify $E p h \times 2$-specific pathophysiological responses to pressure overload in wild-type but not in $E p h \times 2$ knockout mice.

Telemetric blood pressure recordings showed a similar temporal blood pressure increase in both groups (Fig. 6a). As expected from our rat experiments, in which the SHHF allele was associated with high Epxh2 expression and reduced ejection fraction, we found a substantially decreased ejection fraction after 3 weeks of Ang II infusion in wild-type mice compared to knockout mice (Fig. 6b,c).

Cardiac arrhythmias are common features and a principal cause of death in the failing human heart ${ }^{26}$. We therefore tested whether Ephx 2 gene ablation protected against Ang IIinduced arrhythmias. The ventricular refractory period was noticeably prolonged in Ephx2 
knockout mice compared to wild-type mice (Fig. 7a), supporting a prolonged-that is, beneficial—cardiac repolarization in Ephx 2 knockout mice. Notably, there were spontaneous supraventricular and premature ventricular contractions in wild-type mice that were not observed in Ephx 2 knockout mice (Fig. 7b). We then applied programmed electrical stimulations and induced supraventricular tachycardia and atrial fibrillation, as well as short episodes of non-sustained ventricular tachycardia, in all wild-type mice, whereas $E p h \times 2$ knockout mice had stable sinus rhythm even under isoproterenol challenge (Fig. 7c). These findings were corroborated using an independent pressure overload model using thoracic aortic banding (Supplementary Note and Supplementary Fig. 6 online).

\section{$E P H X 2$ expression is decreased in humans with heart failure}

If an imbalance of EET concentrations is critical to the pathogenesis of heart failure, we would expect transcriptional regulation of Ephx2 to maintain high EET levels to be a general defense mechanism, irrespective of the trigger eliciting heart failure. To test this hypothesis, we determined cardiac $E P H X 2$ expression in biopsies from subjects with ischemic heart failure and from controls. Cardiac EPHX2 expression was reduced by $61.5 \%\left(P^{1 / 4} 0.025\right)$ in subjects with heart failure, compared to that in controls (Fig. 8). This finding supports transcriptional downregulation of $E P H X 2$ as a potentially beneficial adaptive mechanism in human heart failure. This hypothesis is supported by the fact that wild-type mice that developed a reduced ejection fraction under two independent models of pressure overload conditions (chronic Ang II infusion and aortic banding) showed a significant downregulation of Ephx2 (Supplementary Note and Supplementary Fig. 7 online).

\section{DISCUSSION}

In humans, hypertension associated with sustained cardiac hypertrophy represents one of the most common causes of heart failure ${ }^{27,28}$. Cardiomyocyte hypertrophy is a primary adaptive response to diminish wall stress induced by mechanical overload. However, the resultant LVH is clinically associated with increased morbidity and mortality ${ }^{2}$. So far, the genetic and molecular checkpoints gating the transition from hemodynamic overload to maladaptive cardiac remodeling and heart failure are poorly understood ${ }^{29,30}$. Recent data suggest a substantial contribution of familial factors that could account for as much as $70 \%$ increased risk for heart failure, after adjustments for other risk factors including $\mathrm{LVH}^{7}$.

We present evidence that genetic variation in Ephx 2 facilitates progression from hypertension and cardiac hypertrophy to heart failure in a rat model of human hypertension and heart failure. We first generated experimental $F_{2}$ intercrosses of SHHF with SHRSP and WKY as reference strains with different set points regarding blood pressure and LVH. Hemodynamic characteristics of parental rat strains were in line with already established echocardiographic and hemodynamic data for rats of strains $\mathrm{SHHF}^{24}$ and $\mathrm{SHR}^{31}$, a close relative of the SHRSP. We documented marked cardiac hypertrophy in SHHF rats, together with decreased left ventricular ejection fraction, indicating the expected alteration of systolic function. By contrast, SHRSP rats retain systolic function, as documented by others ${ }^{31-34}$ and as we have demonstrated here. Thus, we used SHRSP, a strain that is genetically closely related to SHHF, as a reference model. However, rats of our candidate strain, SHHF, clearly 
demonstrated decreased ejection fraction and other heart failure features also common to humans. Linkage analysis to left ventricular hemodynamic parameters of cardiac function in experimental SHHF crosses with WKY and SHRSP revealed significant segregation of ejection fraction with a QTL on rat chromosome 15 harboring Ephx2. The fact that significant linkage of the Ephx 2 locus to left ventricular ejection fraction was detected in the $\mathrm{F}_{2}(\mathrm{SHHF} \times \mathrm{SHRSP})$ cross but not in the $\mathrm{F}_{2}(\mathrm{SHHF} \times \mathrm{WKY})$ cross underscores the pathophysiological importance of hypertension and LVH as permissive factors in deteriorating cardiac function. In human heart failure, a decreased ejection fraction and clinical symptoms of heart failure are preceded by structural and hemodynamic abnormalities, such as increased LVEDV and reduced contractility ${ }^{35}$. We identified these phenotypes in $\mathrm{F}_{2}$ populations; both traits significantly segregated with the Ephx2 locus when both crosses were analyzed jointly (data not shown), suggesting a common regulation of hemodynamic responses to pressure overload by the chromosome 15 QTL. Our genomewide linkage analysis revealed two more significant QTLs, on chromosome 8 and chromosome 4 , for ejection fraction and associated hemodynamic traits in the $\mathrm{F}_{2}$ (SHHF $\times$ SHRSP) population (J.F., J.M., S.P., H.S., M.H. et al., unpublished data). Further characterization of these loci, together with the eQTL data obtained, may generate additional insights into the genetic factors contributing to heart failure in this model.

We performed linkage analysis of cardiac gene transcription as intermediate phenotypes and identified Ephx2 as a prime candidate gene within the heart failure chromosome 15 QTL due to its highly significant cis regulation and its expression pattern across parental strains. Eph 2 2 encodes an enzyme responsible for the conversion of EETs to their corresponding diols $^{36}$, thus diminishing their biological activity ${ }^{37-39}$. We were aided by the recent development of pharmacological Ephx 2 inhibitors. These experiments showed that EETs elicit a number of cardioprotective actions including vasodilation, anti-inflammatory effects, endothelial cell proliferation, antiaggregatory effects and inhibition of vascular smooth muscle cell migration ${ }^{25}$. In addition, recent studies have shown an increased availability of cardioprotective EETs in Ephx 2 knockout mice with associated benefits on left ventricular function due to reduced infarction size after ischemic injury ${ }^{40}$. Similar to our own observations, baseline cardiac anatomy and contractile function in this study were not different in Ephx 2 knockout and wild-type mice, suggesting that detrimental effects of environmental stress to the heart in general (ischemia or pressure overload) can be counteracted by a decreased action of Ephx 2 together with the maintenance of high EET levels.

The Eph $x 2$ locus accounted for almost two-thirds of the Ephx 2 expression variability in a large proportion in the $\mathrm{F}_{2}(\mathrm{SHHF} \times \mathrm{SHRSP})$ population. However, the association of the Eph $x 2$ locus with ejection fraction as a far more complex phenotype was smaller in magnitude. Overall, the Ephx 2 locus accounted for about $15 \%$ of the phenotypic variance for ejection fraction. We demonstrated that Ephx 2 transcript levels significantly correlate with the tails of the distribution for ejection fraction, providing independent evidence that the allelic modulation of Eph $\times 2$ transcript levels plays a causal role in the development of heart failure. Rats with high transcript levels had lower ejection fractions, whereas rats with lower transcript levels had higher ejection fractions. It is important to note that allelic variants of $E p h \times 2$ appear to impact heart failure as a complex disease through perturbations 
of trans-regulated gene networks, and that transcriptional phenotypes are again intermediate to the clinically defined disease. We showed that transcriptional regulation not only of Ephx 2 but also of several other genes in the entire Ephx2 pathway are affected by the chromosome 15 QTL in our model. We applied pathway linkage scores to assess the statistical significance of this genetic effect and found a genome-wide significant association of expression on a pathway level with the maximum effect at the Ephx2 locus.

To uncover DNA variants that can account for the observed alterations of Ephx2 expression and function, we performed comparative sequence analysis of $E p h \times 2$ in heart failure and reference strains. We detected a regulatory insertion/deletion polymorphism within the AP-1 binding site and showed enhanced promoter activity in SHHF. However, our findings do not preclude that other regulatory variants contribute to the allele-specific differences in Ephx 2 expression besides this regulatory insertion/deletion polymorphism in AP-1. Since AP-1 activation has also been demonstrated in human heart failure ${ }^{41}$, and Ang II serves to increase Ephx2 expression through AP-1 activation ${ }^{42}$, it seems possible that EPHX2 promoter variants affecting AP-1 binding may also constitute a contributory mechanism for heart failure susceptibility in humans. It would be interesting to test variation at $E P H X 2$ for association with human hypertensive heart failure.

Eph $\times 2$ was established as a primary heart failure candidate gene by combined evidence from linkage analyses, global $F_{2}$ transcription profiling, sequence analysis and functional data on protein abundance and Ephx 2 enzymatic activity. Additional experimental support for the specific relevance of Ephx2 in the genesis of hypertension-induced heart failure was generated by experiments in mice with targeted disruption of Ephx2. In keeping with previous studies in two independently-derived colonies of Ephx 2 knockout mice, including the one used in our experiment ${ }^{43}$, we found no differences in blood pressure and ejection fraction between knockout and wild-type mice at baseline. However, after exposing both strains to pressure-overload conditions, Ephx 2 knockout mice showed only about half of the ejection fraction decline seen in wild-type mice, although blood pressure increases to high and prolonged dosages of Ang II were similar in both strains. This situation resembles the SHHF model, which develops heart failure while its equally hypertensive cousin, the SHRSP, does not. Furthermore, hypertensive Ephx 2 knockout mice are protected from cardiac arrhythmias. This is in agreement with findings using pharmacological inhibition of Ephx2 (ref. 44) and may be explained by previous observations suggesting that EETs are modulators of ion channel function in cardiomyocytes (for review, see ref. 25).

We are the first to present evidence that $E p h \times 2$ is a heart failure susceptibility gene. Ephx2 allelic variability leads to quantitative differences in transcript levels that in turn quantitatively regulate the metabolism of cardioprotective epoxyeicosatrienoic acids. The implications of our findings are important as $E p h \times 2$ has already found attention as a pharmacological target for cardiovascular disease ${ }^{44}$. In light of elevated $E p h \times 2$ expression levels associated with the development of heart failure in rats, and protection from heart failure in $E p h \times 2$-deficient mice, we interpret $E P H X 2$ downregulation in human failing hearts as an adaptive transcriptional process aimed at maintaining high EET levels. EETs are known to exert cardioprotective actions ${ }^{45}$. Conversely, individuals with genetically determined, persistently high cardiac EPHX2 expression, together with arterial hypertension 
and LVH, probably represent a high-risk group for disease progression to heart failure. The role we have shown for Ephx 2 in the complex initiation of the disease in rats and mice, as well as its regulation in human heart failure, suggest a potential avenue for developing new heart failure treatments.

\section{METHODS}

\section{Experimental crosses and mice.}

SHHF/Bbb, WKY/Bbb and SHRSP/Bbb rats were from our colonies at the Max-Delbrück Center for Molecular Medicine and were maintained under strict inbreeding. SHHF breeders (SHHF/Mcc) were initially obtained from the original colonies of S.A. McCune (Department of Integrative Physiology, University of Colorado at Boulder). SHHF were mated with SHRSP and WKY, respectively, to generate $F_{1}$ progenies. $F_{1}$ rats for each cross were brother-sister mated, resulting in $166 \mathrm{~F}_{2}(\mathrm{SHHF} \times \mathrm{SHRSP})(87$ male and 79 female) and $189 \mathrm{~F}_{2}(\mathrm{SHHF} \times \mathrm{WKY})(93$ male and 96 female) rats. All rats were investigated at 14 months of age. Ephx $2^{-/-}$mice were previously described ${ }^{43}$ and were further backcrossed for nine generations onto $\mathrm{C} 57 \mathrm{BL} / 6 \mathrm{ByJ}$ before used in our studies. Heterozygote $\mathrm{F}_{9}$ mice were crossed to obtain homozygote null and wild-type littermates used in this study. All mouse experiments were performed starting at 16 weeks of age; only male mice were used. All animal experiments were conducted according to the guidelines of the American Physiological Society and with approval of the animal protection authorities of the state of Berlin (Landesamt Für Gesundheit und Soziales).

\section{Hemodynamic measurements.}

Rats were intubated and ventilated under isoflurane anesthesia. A 2-French conductance catheter (SPR 838 Aria, Millar Instruments) was inserted into the left ventricle through the right carotid artery. Left ventricular contractility was obtained from the ventricular pressure curves. Ventricular volumes were determined as described ${ }^{46}$.

\section{Telemetry.}

Telemetric blood pressure measurements were performed as previously described ${ }^{47}$. The data from the TA11PA-C20 device were sampled every $5 \mathrm{~min}$ for $10 \mathrm{~s}$ continuously day and night with a sampling rate of $1,000 \mathrm{~Hz}$. Mice were allowed to recover for three days before baseline recording started. SBP, DBP and HR were recorded using the DATAQUEST software.

\section{Echocardiography.}

Echocardiography was performed before and after 3 weeks of Ang II treatment as previously described ${ }^{48}$. Briefly, mice were anesthetized with $2 \%$ isoflurane and kept warm on a heated platform. Temperature and ECG were continuously monitored. Cardiac function and morphology were assessed by echocardiography with a VisualSonics Vevo 770 HighResolution Imaging System with the use of a high-resolution (40 MHz) transducer. 


\section{Surface ECG and in vivo electrophysiology studies.}

Each mouse was slightly anaesthetized with $1.6 \%$ isoflurane and surface Electrocardiograms (ECG) were obtained using adhesive electrodes on each limb after 3 weeks of Ang II treatment. ECG standard intervals were measured in six standard leads. In vivo electrophysiological studies (EPS) with programmed electrical stimulations (PES) were performed at baseline and after isoproterenol treatment (intraperitoneal injection of $0.02 \mathrm{mg}$ isoproterenol/g body weight). Briefly, an octapolar 2-French electrode catheter (CIBer mouse cath, NuMed Inc.) was placed via the right jugular vein into the right atrium and ventricle under electrogram guidance. Electrical stimuli were delivered at twice diastolic threshold with a pulse duration of $1.0 \mathrm{~ms}$. PES used up to three extra stimuli after 10 basal stimuli (cycle length 90/100/120 ms). Additionally, short episodes of burst pacing (10 s) with cycle lengths down to $60 \mathrm{~ms}$ were applied. Only reproducible arrhythmias of three beats or more were considered. All electrograms were digitally stored for off-line analysis.

\section{Marker selection and genotyping.}

SNPs were identified by the STAR consortium and are publicly available at http:// www.ensembl.org. SNP genotyping in $\mathrm{F}_{2}$ crosses was carried out using the GoldenGate genotyping platform (Illumina Inc., San Diego). Microsatellite markers were genotyped using fluorescence-based semi-automated genotyping on an ABI3730 sequencer. SNP and microsatellite genotyping results are reported in Supplementary Table 2 online. Statistical methods for genetic mapping and linkage analysis are reported in Supplementary Methods online.

\section{Preparation of labeled cDNA and hybridizations to microarrays.}

We pulverized whole frozen hearts and stored aliquots at $-801 \mathrm{C}$ for RNA and $-201 \mathrm{C}$ for protein experiments. Total RNA was extracted using Trizol reagent (Invitrogen) and purified using RNeasy Mini kit (Qiagen) in accordance with the manufacturer's protocol. Doublestranded cDNA was synthesized from total RNA using the One-Cycle cDNA Synthesis Kit (Affymetrix) and cRNA biotinylated from cDNA using the IVT Labeling Kit (Affymetrix). We hybridized the fragmented cRNA samples to Affymetrix Rat Genome 2302.0 arrays in accordance with the Affymetrix protocol. Statistical methods for eQTL analysis are reported in Supplementary Methods.

\section{Real-time RT-PCR.}

We reverse-transcribed total RNA with oligo(dT) primers (Gibco-BRL) and Superscript II reverse transcriptase (Invitrogen). We designed primers and probes using Primer Express 2.0 (Applied Biosystems) and placed them in non-polymorphic regions of the rat Ephx2 gene (Supplementary Table 3 online). For human TaqMan probes, Applied Biosystems assay (hs00157403_m1) was used. TaqMan analysis was performed using an Applied Biosystems $7900 \mathrm{HT}$ system. We normalized expression levels to GAPDHRNA expression by using the $2^{-} \mathrm{DD}^{\mathrm{CT}}$ method. 


\section{Analysis of Ephx2 hydrolase activity.}

Hydrolase activities were determined by using $10 \mathrm{mg}$ of heart-extracted proteins $(10,000 \mathrm{~g}$ supernatant) dissolved in $100 \mathrm{mM}$ potassium phosphate buffer, $\mathrm{pH}$ 7.2. Proteins were incubated with $10 \mathrm{mM}\left[1-{ }^{14} \mathrm{C}\right] 14,15$-EET for $5 \mathrm{~min}$ at $37 \mathrm{lC}$. Adding ethyl acetate terminated the reaction. After extraction, products were evaporated under nitrogen and dissolved in ethanol. Metabolites were resolved by reverse-phase HPLC (Shimadzu LC 10 Avp). Retention times for 14,15-EET/-DHET were 23.4 and $12.7 \mathrm{~min}$, respectively. Methods for protein blot analyses and production of recombinant proteins are reported in Supplementary Methods.

\section{Promoter activity assays.}

The -2079 to +41 bp Ephx 2 promoter was cloned into a pGL3-Basic vector (Promega) containing the firefly luciferase gene as a reporter encompassing either the SHHF or the SHRSP promoters, and integrity was confirmed after cloning by sequence analysis. Since SHRSP and WKY share common haplotypes, we cloned only the promoter variants from one of the strains (SHRSP) and tested the variants against the SHHF promoter. For transient transfections, H9c2 (ATCC) cells were cotransfected with pRL-SV40 plasmid (Promega) as a normalizing control. All transfections were carried out in triplicates. Firefly and Renilla luciferase activity was analyzed with the Dual-Luciferase Reporter Assay System according to manufacturer's description (Promega).

\section{Electrophoretic mobility shift assay.}

Binding of AP-1 to the Ephx 2 promoter in vitro was carried out as previously described ${ }^{49}$. Briefly, synthetic double-stranded and $3 \notin$ biotin-labeled oligonucleotides and recombinant protein (Promega) were incubated at room temperature for 20 min using the LightShift Chemiluminescent EMSA Kit (Pierce). The binding reaction contained $\mathrm{x} 1$ binding buffer, $0.01 \mathrm{mg} / \mathrm{ml}$ poly(dI:dC), $0.05 \% \mathrm{NP}-40,50 \mathrm{mM} \mathrm{KCl}, 0.5 \mathrm{mM} \mathrm{MgCl} 2$ and $0.5 \mathrm{mM}$ DTT. Two-hundred-fold molar excesses of unlabeled oligonucleotides were used as competitors. Reaction mixtures were separated with 5\% polyacrylamide gels. Probes were transferred to a positively charged nylon membrane (Ambion), UV-cross-linked and detected by stabilized Streptavidin-Horseradish Peroxidase Conjugate (Pierce).

\section{Quantification of EETs in the heart.}

Heart tissue was homogenized using a biopulverizer (Biospec Products). $40 \mathrm{mg}$ aliquots were subjected to alkaline hydrolysis, followed by a solid phase extraction. Quantification of EETs in the heart homogenates was done by the Lipidomix GmbH, Berlin, using HPLC (Agilent 1200) with tandem mass spectroscopy Triple Quad MS/MS (Agilent 6410). Detection limit for EETs was $0.2 \mathrm{ng}$ per gram of tissue.

\section{Human myocardial biopsies for mRNA expression analysis.}

Full thickness myocardial biopsies were harvested at the time of cardiac surgery with the approval of the Hammersmith Hospital Research Ethics Committee. Heart function was characterized by trans-thoracic echocardiography. Individuals with coronary artery disease but having normal ventricular function were used as controls. Individuals with heart failure 
were defined as an ejection fraction of $045 \%$ secondary to ischemic heart disease. Written informed consent was obtained from all subjects.

\section{Supplementary Material}

Refer to Web version on PubMed Central for supplementary material.

\section{ACKNOWLEDGMENTS}

We thank A. Müller, H. Kistel, A. Schiche, J. Mothes, J. Meisel, M. Rothe and M. Taube for technical assistance. We acknowledge funding to N.H. from the German Ministry for Science and Education (National Genome Research Network) and support through STAR and EURATools (European Commission contract LSHGCT-2004-005235 and LSHG-CT-2005-019015); to F.C.L. and W.-H.S. from the Deutsche Forschungsgemeinschaft; to B.D.H. from US National Institute of Environmental Health Sciences grant R37 ES02710; and to S.A.C. from the British Heart Foundation and the UK Department of Health.

\section{References}

1. Kannel WB \& Belanger AJ The epidemiology of heart failure. Am. Heart J 121, 951-957 (1991). [PubMed: 2000773]

2. Levy D et al. Long-term trends in the incidence of and survival with heart failure. N. Engl. J. Med 347, 1397-1402 (2002). [PubMed: 12409541]

3. Owan TE et al. Trends in prevalence and outcome of heart failure with preserved ejection fraction. N. Engl. J. Med 355, 251-259 (2006). [PubMed: 16855265]

4. Chien KR Genomic circuits and the integrative biology of cardiac diseases. Nature 407, 227-232 (2000). [PubMed: 11001065]

5. Kostis JB et al. Prevention of heart failure by antihypertensive drug treatment in older persons with isolated systolic hypertension. SHEP Cooperative Research Group. J. Am. Med. Assoc 278, $212-$ 216 (1997).

6. Dahlof B et al. Morbidity and mortality in the Swedish Trial in Old Patients with Hypertension (STOP-Hypertension). Lancet 338, 1281-1285 (1991). [PubMed: 1682683]

7. Lee DS et al. Association of parental heart failure with risk of heart failure in offspring. N. Engl. J. Med 355, 138-147 (2006). [PubMed: 16837677]

8. McCune S, Baker PB \& Stills FH SHHF/Mcc-cp rat: model of obesity, non-insulin-dependent diabetes, and congestive heart failure. Ilar News 32, 23-27 (1990).

9. Sack MN et al. Fatty acid oxidation enzyme gene expression is downregulated in the failing heart. Circulation 94, 2837-2842 (1996). [PubMed: 8941110]

10. Heyen JR et al. Structural, functional, and molecular characterization of the SHHF model of heart failure. Am. J. Physiol. Heart Circ. Physiol 283, H1775-H1784 (2002). [PubMed: 12384454]

11. Holycross BJ, Summers BM, Dunn RB \& McCune SA Plasma renin activity in heart failure-prone SHHF/Mcc-facp rats. Am. J. Physiol 273, H228-H233 (1997). [PubMed: 9249494]

12. Bergman MR, Kao RH, McCune SA \& Holycross BJ Myocardialtumor necrosis factor-a secretion in hypertensive and heart failure-prone rats. Am. J. Physiol 277, H543-H550 (1999). [PubMed: 10444479]

13. Brem RB, Yvert G, Clinton R \& Kruglyak L Genetic dissection of transcriptional regulation in budding yeast. Science 296, 752-755 (2002). [PubMed: 11923494]

14. Grupe A et al. In silico mapping of complex disease-related traits in mice. Science 292, 1915-1918 (2001). [PubMed: 11397946]

15. Karp CL et al. Identification of complement factor 5 as a susceptibility locus for experimental allergic asthma. Nat. Immunol 1, 221-226 (2000). [PubMed: 10973279]

16. Klose J et al. Genetic analysis of the mouse brain proteome. Nat. Genet 30, 385-393 (2002). [PubMed: 11912495] 
17. Liao $\mathrm{G}$ et al. In silico genetics: identification of a functional element regulating $\mathrm{H} 2$-Ea gene expression. Science 306, 690-695 (2004). [PubMed: 15499019]

18. Monks SA et al. Genetic inheritance of gene expression in human cell lines. Am. J. Hum. Genet 75, 1094-1105 (2004). [PubMed: 15514893]

19. Morley $\mathrm{M}$ et al. Genetic analysisofgenome-wide variation inhuman geneexpression. Nature 430, 743-747 (2004). [PubMed: 15269782]

20. Schadt EE et al. Genetics of gene expression surveyed in maize, mouse and man. Nature 422, 297302 (2003). [PubMed: 12646919]

21. Bystrykh L et al. Uncovering regulatory pathways that affect hematopoietic stem cell function using 'genetical genomics'. Nat. Genet 37, 225-232 (2005). [PubMed: 15711547]

22. Chesler EJ et al. Complex trait analysis of gene expression uncovers polygenic and pleiotropic networks that modulate nervous system function. Nat. Genet 37, 233-242 (2005). [PubMed: 15711545]

23. Hübner $\mathrm{N}$ et al. Integrated transcriptional profiling and linkage analysis for identification of genes underlying disease. Nat. Genet 37, 243-253 (2005). [PubMed: 15711544]

24. Reffelmann T \& Kloner RA Transthoracic echocardiography in rats. Evalution of commonly used indices of left ventricular dimensions, contractile performance, and hypertrophy in a genetic model of hypertrophic heart failure (SHHF-Mcc-facp-Rats) in comparison with Wistar rats during aging. Basic Res. Cardiol 98, 275-284 (2003). [PubMed: 12955400]

25. Imig JD Cardiovascular therapeutic aspects of soluble epoxide hydrolaseinhibitors. Cardiovasc. Drug Rev 24, 169-188 (2006). [PubMed: 16961727]

26. Solomon SD et al. Influence of ejection fraction on cardiovascular outcomes in a broad spectrum of heart failure patients. Circulation 112, 3738-3744 (2005). [PubMed: 16330684]

27. Molkentin JD \& Dorn GW II. Cytoplasmic signaling pathways that regulate cardiac hypertrophy. Annu. Rev. Physiol 63, 391-426 (2001). [PubMed: 11181961]

28. Frey N, Katus HA, Olson EN \& Hill JA Hypertrophy of the heart: a new therapeutic target? Circulation 109, 1580-1589 (2004). [PubMed: 15066961]

29. Seidman JG \& Seidman C The genetic basis for cardiomyopathy: from mutation identification to mechanistic paradigms. Cell 104, 557-567 (2001). [PubMed: 11239412]

30. Watkins H \& Farrall M Genetic susceptibility to coronary artery disease: from promise to progress. Nat. Rev. Genet 7, 163-173 (2006). [PubMed: 16462853]

31. Pfeffer MA \& Frohlich ED Hemodynamic and myocardial function in young and old normotensive and spontaneously hypertensive rats. Circ. Res 32(suppl. 1), 28-38 (1973).

32. Cingolani OH, Yang XP, Cavasin MA \& Carretero OA Increased systolic performance with diastolic dysfunction in adult spontaneously hypertensive rats. Hypertension 41, 249-254 (2003). [PubMed: 12574090]

33. Shorofsky SR et al. Cellular mechanisms of altered contractility in the hypertrophied heart: big hearts, big sparks. Circ. Res 84, 424-434 (1999). [PubMed: 10066677]

34. Slama M, Ahn J, Varagic J, Susic D \& Frohlich ED Long-term left ventricular echocardiographic follow-up of SHR and WKY rats: effects of hypertension and age. Am. J. Physiol. Heart Circ. Physiol 286, H181-H185 (2004). [PubMed: 12958037]

35. Hunt SA et al. ACC/AHA guidelines for the evaluation and management of chronic heart failure in the adult: executive summary. A report of the American College of Cardiology/American Heart Association Task Force on Practice Guidelines (Committee to revise the 1995 Guidelines for the Evaluation and Management of Heart Failure). J. Am. Coll. Cardiol 38, 2101-2113 (2001). [PubMed: 11738322]

36. Hammock BD, Ratcliff M \& Schooley DA Hydration of an 180 epoxide by a cytosolic epoxide hydrolase from mouse liver. Life Sci 27, 1635-1641 (1980). [PubMed: 7442462]

37. Imig JD, Zhao X, Capdevila JH, Morisseau C \& Hammock BD Soluble epoxide hydrolase inhibition lowers arterial blood pressure in angiotensin II hypertension. Hypertension 39, 690-694 (2002). [PubMed: 11882632]

38. Spector AA, Fang X, Snyder GD \& Weintraub NL Epoxyeicosatrienoic acids (EETs): metabolism and biochemical function. Prog. Lipid Res 43, 55-90 (2004). [PubMed: 14636671] 
39. Spiecker M \& Liao JK Vascular protective effects of cytochrome p450 epoxygenase-derived eicosanoids. Arch. Biochem. Biophys 433, 413-420 (2005). [PubMed: 15581597]

40. Seubert JM et al. Roleofsolubleepoxide hydrolase in postischemic recovery of heart contractile function. Circ. Res 99, 442-450 (2006). [PubMed: 16857962]

41. Frantz $S$ et al. Sustained activation of nuclear factor kappa B and activator protein 1 in chronic heart failure. Cardiovasc. Res 57, 749-756 (2003). [PubMed: 12618236]

42. Ai D et al. Angiotensin II up-regulates soluble epoxide hydrolase in vascular endothelium in vitro and in vivo. Proc. Natl. Acad. Sci. USA 104, 9018-9023 (2007). [PubMed: 17495027]

43. Luria A et al. Compensatory mechanism for homeostatic blood pressure regulation in Ephx 2 genedisrupted mice. J. Biol. Chem 282, 2891-2898 (2007). [PubMed: 17135253]

44. Xu D et al. Prevention and reversal of cardiac hypertrophy by soluble epoxide hydrolase inhibitors. Proc. Natl. Acad. Sci. USA 103, 18733-18738 (2006). [PubMed: 17130447]

45. Seubert $\mathrm{J}$ et al. Enhanced postischemic functional recovery in $C Y P 2 J 2$ transgenic hearts involves mitochondrial ATP-sensitive K+ channels and p42/p44 MAPK pathway. Circ. Res 95, 506-514 (2004). [PubMed: 15256482]

46. Baan J et al. Continuous measurement of left ventricular volume in animals and humans by conductance catheter. Circulation 70, 812-823 (1984). [PubMed: 6386218]

47. Gross V et al. Autonomic nervous system and blood pressure regulation in RGS2-deficient mice. Am. J. Physiol. Regul. Integr. Comp. Physiol 288, R1134-R1142 (2005). [PubMed: 15661972]

48. Baurand A et al. b-catenin downregulation is required for adaptive cardiac remodeling. Circ. Res 100, 1353-1362 (2007). [PubMed: 17413044]

49. Fiala-Beer E, Lee AC\& Murray M Regulation of the rat CYP4A2 gene promoter by c-Jun and octamer binding protein-1. Int. J. Biochem. Cell Biol 39, 1235-1247 (2007). [PubMed: 17481938] 


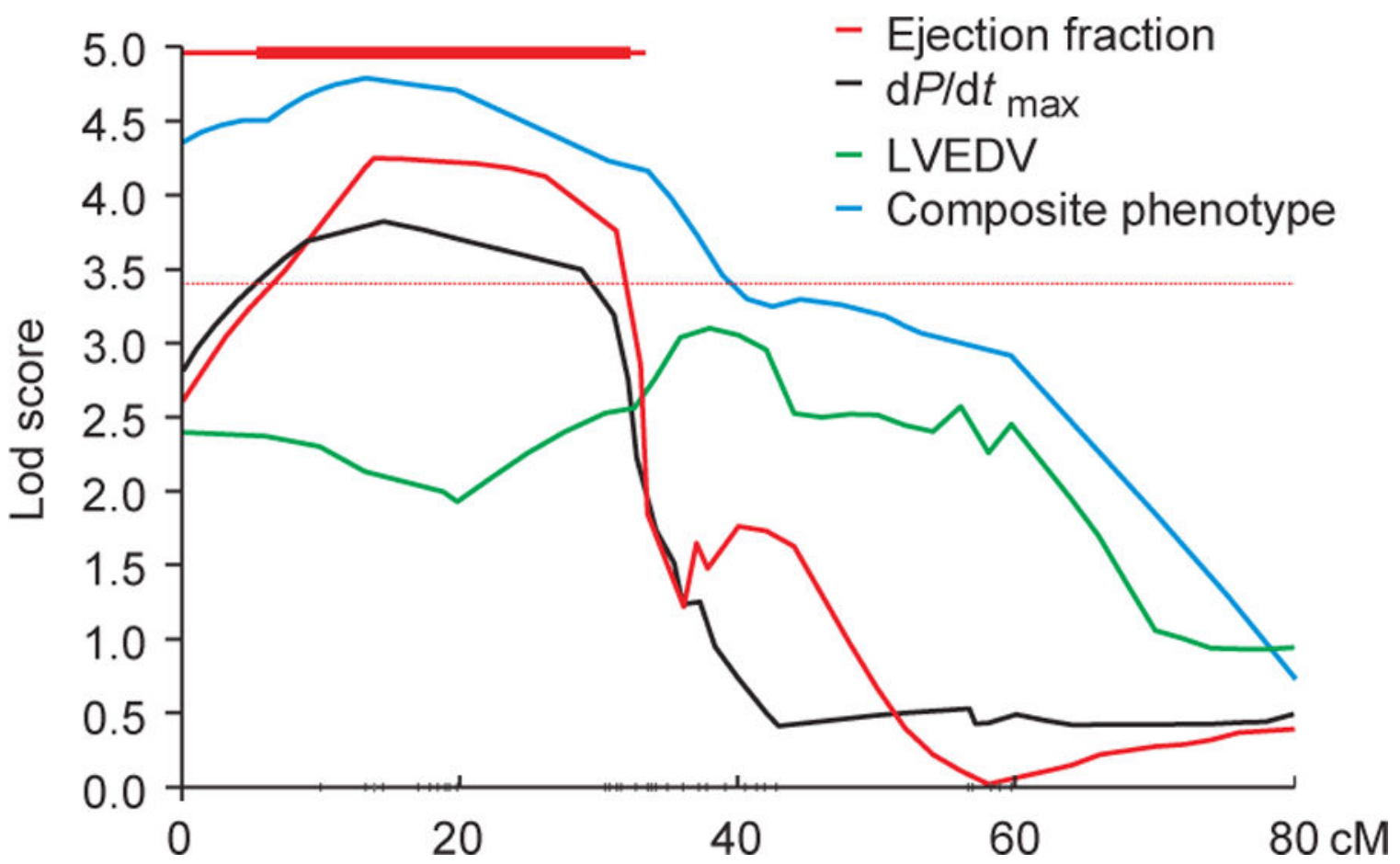

Figure 1.

Colocalization of cardiac phenotype QTL on chromosome 15. Multipoint lod plots in $\mathrm{F}_{2}(\mathrm{SHHF} \times \mathrm{SHRSP})$ for ejection fraction, $\mathrm{d} P / \mathrm{d} t_{\max }, \mathrm{LVEDV}$. Lod scores are plotted against genetic map distances in cM. Gray marks on $x$ axis represent marker positions. Genomewide corrected significance levels $\left(P^{1 / 4} 0.05\right)$ were determined by 1,000 permutations. The dashed horizontal line indicates genome-wide significance for ejection fraction linkage; the thick and thin solid red bar indicates 1- and 2-lod-unit drop confidence interval, respectively. Significance levels for the other traits reported are slightly different and are not depicted for clarity (genome-wide significance at lod 3.2 and 3.5 for $\mathrm{d} P / \mathrm{d} t_{\max }$ and LVEDV, respectively). The composite trait variable constructed from the three traits notably increased the significance of the findings. 


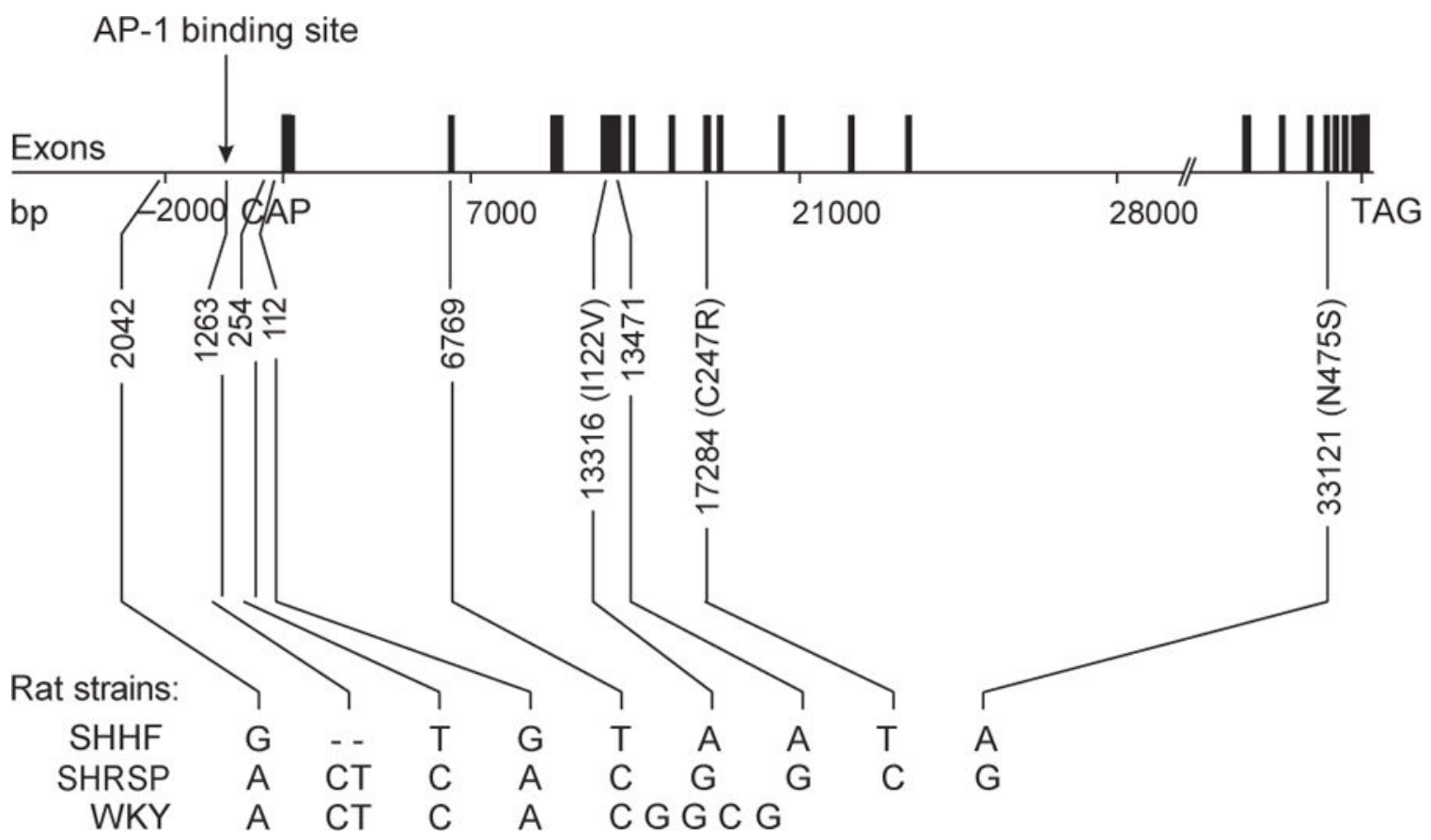

Figure 2.

Polymorphisms in Ephx 2 regulatory and coding region. Four polymorphisms were identified in the putative promoter region, of which a 2-nt insertion in SHRSP and WKY was directly located in an AP-1 binding site (arrow). Five SNPs were detected in Ephx2 exons, three of which led to an amino acid change (marked in parentheses). We found no variation in the $3 \varnothing$ UTR. Exons are pictured as black boxes. In all instances, SHRSP and WKY shared the same allele and haplotype at Ephx2. A variant allele status was observed for SHHF. 

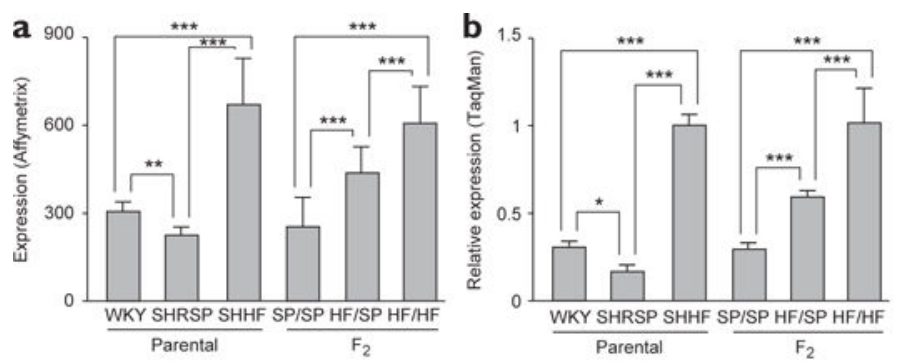

c
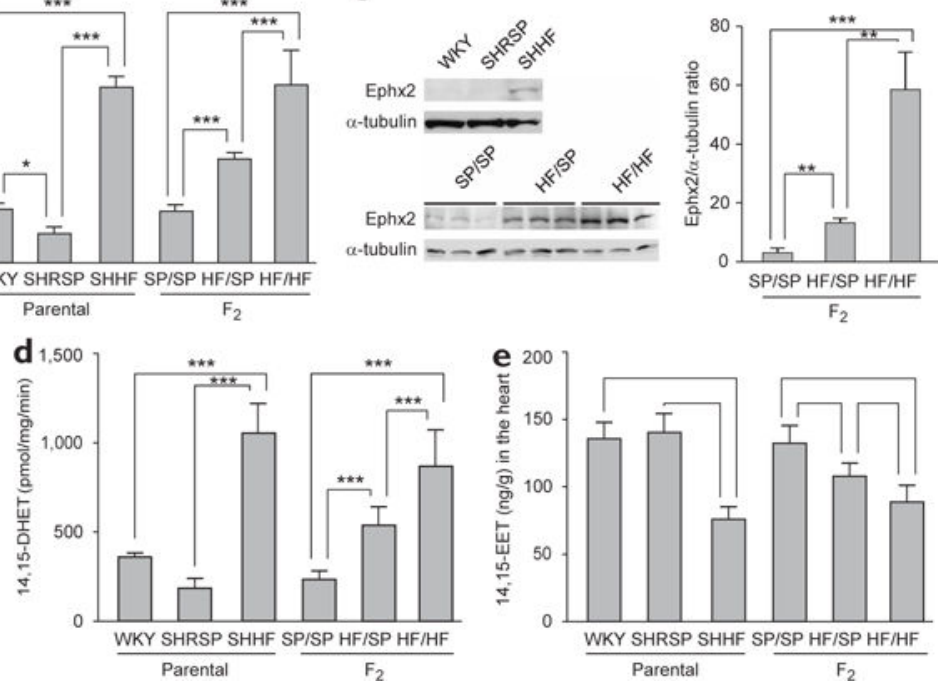

Figure 3.

Ephx 2 expression and sEH activity in heart tissue. (a) Ephx 2 expression measured using microarrays in parental strains ( $n 1 / 45$ per group) and $\mathrm{F}_{2}$ rats according to their genotype at D15Rat10: SHRSP/SHRSP (SP/SP), $n$ 1/4 29; SHHF/SHRSP (HF/SP), $n$ 1/4 76; SHHF/SHHF (HF/HF), $n$ 1/4 32. (b) RT-PCR to confirm microarray expression results; parental strains ( $n^{1 / 4}$ 5 per group) and $\mathrm{F}_{2}$ rats according to their genotype at D15Rat10 (SHRSP/SHRSP $n 1 / 410$; SHHF/SHRSP $n^{1 / 4} 10$; SHHF/SHHF $n^{1 / 4} 10$ ). (c) Representative blot of sEH protein in $\mathrm{F}_{0}$ and $\mathrm{F}_{2}$ rats. Membranes were stripped and rehybridized with an antibody to a-tubulin as a loading control. Quantification of the membrane for $\mathrm{F}_{2}$ rats is shown next to blots. (d) $\mathrm{sEH}$ hydrolase activity per milligram of heart tissue in parental $\left(n \frac{1}{4} 5\right)$ and $\mathrm{F}_{2}$ rats $(n$ 1/4 10 per genotype). Enzymatic activity was determined by measuring the hydrolysis of 14,15 -EET, which is the preferred substrate of $\mathrm{sEH}$ and a prominent metabolite among regioisomeric EET present in rat heart tissue, to 14,15-dihydroxyeicosatrienoic acids (14,15-DHET). (e) 14,15 -EET per gram of heart tissue in parental $\left(n^{1 / 4} 5\right)$ and $\mathrm{F}_{2}$ rats ( $n^{1 / 4} 10$ per genotype).

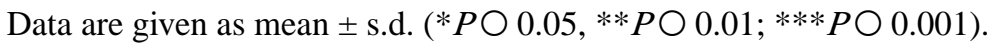


$\mathbf{a}$
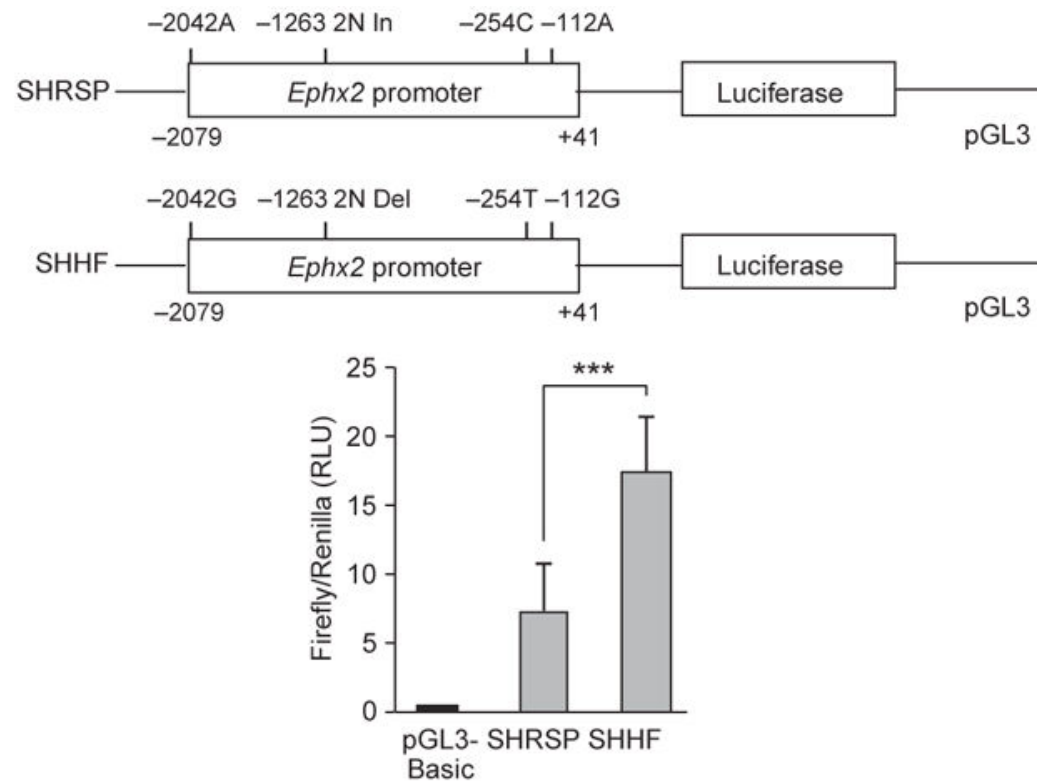

b WKY CCTGAGGCAAGAAGTGGAAGTGTC TGACT CTCAAAAATTAATCCACAGTA

SHRSP CCTGAGGCAAGAAGTGGAAGTGTC TGACT CTCAAAAATTAATCCACAGTA

SHHF CCTGAGGCAAGAAGTGGAAGTGTC TGA EFCAAAAATTAATCCACAGTA

AP- 1 consensulus

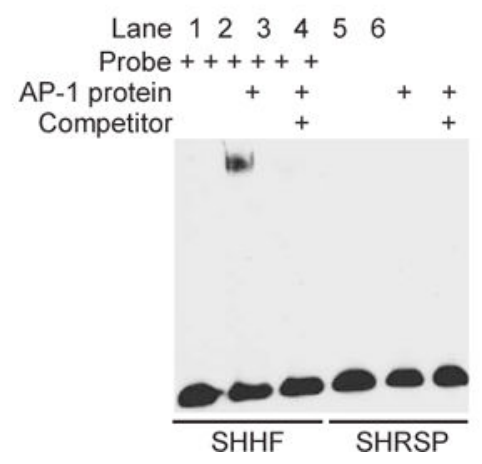

Figure 4.

Promoter polymorphisms of Ephx2 influence promoter activity. (a) Schematics of reporter constructs used for transfection of H9c2 cells and, below, luciferase expression of each construct and empty pGL3-Basic vector in $\mathrm{H} 9 \mathrm{c} 2$ cells. Each value represents mean \pm s.d. of at least three experiments; $* * * P \bigcirc 0.001$. RLU, relative light units. (b) Top, sequence alignment of SHHF, SHRSP and WKY Ephx2 promoter sequences containing the AP-1 binding sequence. Computational analysis predicts that, in SHHF, the AP-1 consensus sequence is completely restored, suggesting that it was intact ancestrally, whereas, in SHRSP and WKY, a 2-bp insertion disrupts AP-1 binding. Bottom, an electrophoretic mobility shift assay with biotin-labeled probes. Lanes 1 and 4, probes only; lanes 2, 3, 5 and 6, probes and AP-1 recombinant protein; lanes 3 and 6, probes, AP-1 recombinant protein and unlabeled competitor. 


\subsubsection{4}

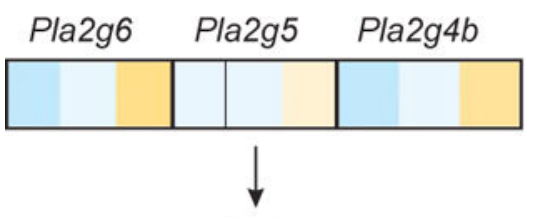

Arachidonic acid

1.14.14.1
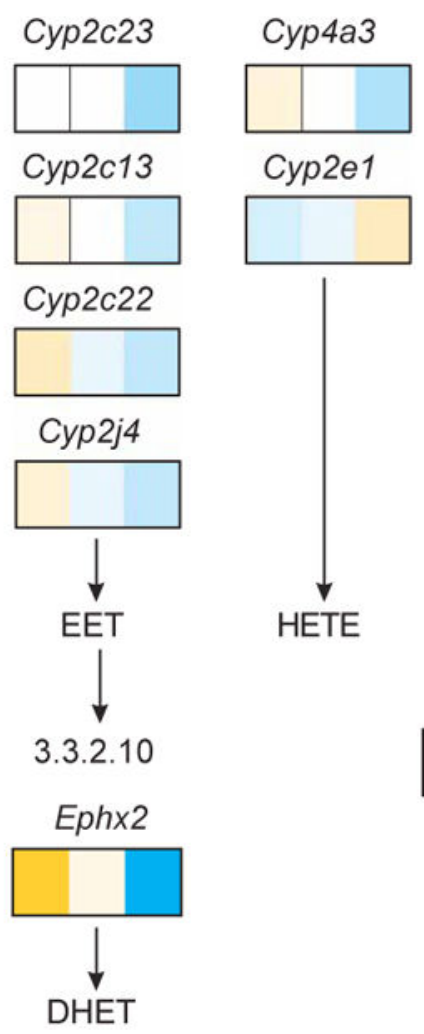

$-1.288$

$\square \quad-1.194$

$\square-1.100$

$\square-1.007$

$\square-0.913$

$\square \quad-0.819$

$\square \quad-0.725$

$\square-0.631$

$\square-0.538$

$\square-0.444$

$\square \quad-0.350$

$\square-0.256$

$\square-0.163$

$-0.069$

0.000

0.025

0.119

0.213

0.306

0.400

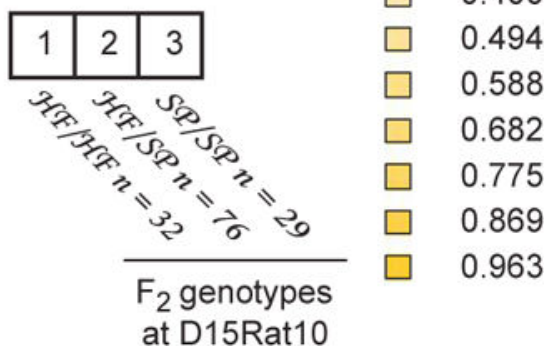

Figure 5.

Identification of regulatory trans eQTLs mapping to chromosome 15. A simplified overview of the Ephx 2 pathway is depicted. Each box is headed by an enzyme classification number describing the reaction that is catalyzed. All genes analyzed with the corresponding enzymatic function are listed in the boxes below. For each gene, a color code indicates the genotype-specific average expression values according to zygosity-SHHF/SHHF (HF/HF), SHHF/SHRSP (HF/SP) and SHRSP/SHRSP (SP/SP), from left to right—at the Ephx2 locus. The expression matrix has been centered and scaled to $\mathrm{s} 1 / 41$ before visualization. Orange, overexpression; white, baseline; cyan, underexpression. CYP genes are subdivided into two columns, with the gene products of those on the left producing predominantly EETs, which are converted to dihydroxyeicosatrienoic acids (DHETs) by $\mathrm{sEH}$, and the right producing predominantly hydroxyeicosatetraenoic acids (HETEs). The Ephx2 pathway has its genomewide pathway linkage peak at the $E p h \times 2$ locus with $P \bigcirc 10^{-6}$. 

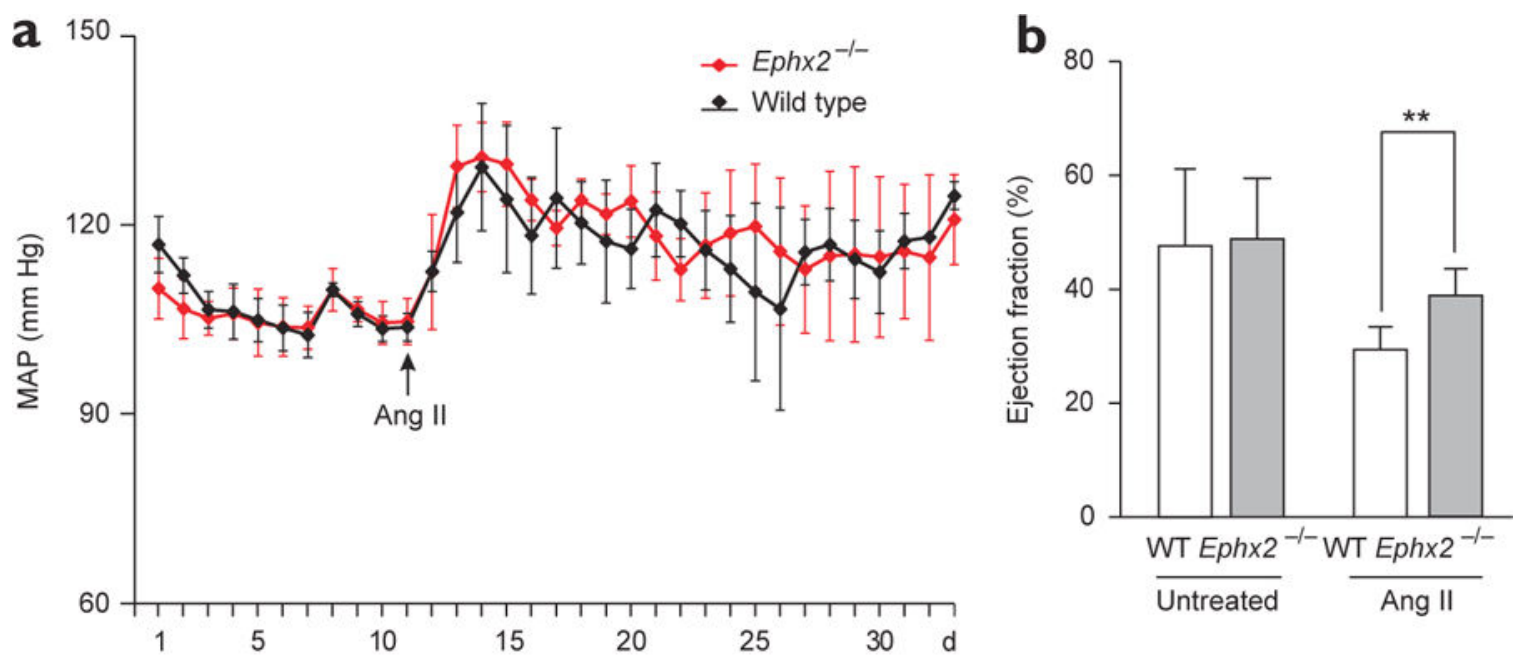

C
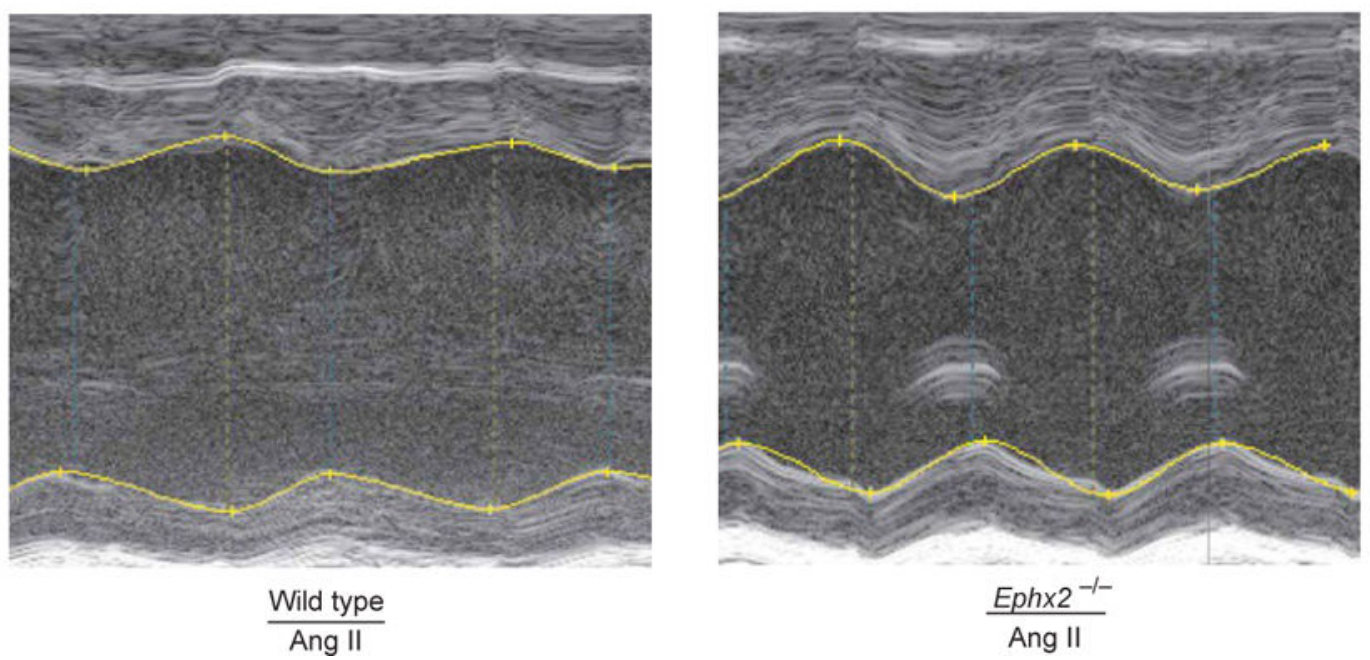

Figure 6.

Characterization of Ephx $2^{-/-}$mice by telemetry and echocardiography. (a) Blood pressure measurements (mean arterial pressure, MAP) in conscious mice (values given as mean \pm s.d.; $n^{1 / 4} 4$ per group). (b) Ejection fraction, measured by echocardiography (mean \pm s.d.), in Eph $\times 2^{-/-}\left(n^{1 / 4} 17\right)$ and wild-type $\left(n^{1 / 4} 15\right)$ mice before and after Ang II treatment; ** $P O$ 0.01. (c) Examples of M-mode echocardiography in Ephx $2^{--}$and wild-type mice after Ang II treatment. Blue vertical lines, left ventricular end-systolic diameter; orange vertical lines, left ventricular end-diastolic diameter. 
a
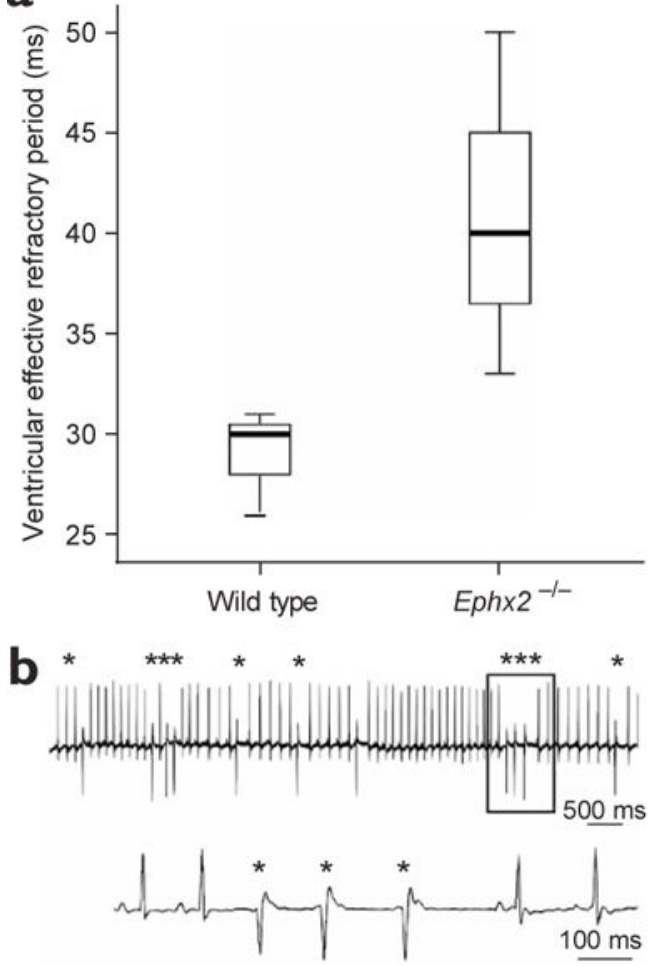

Wild type

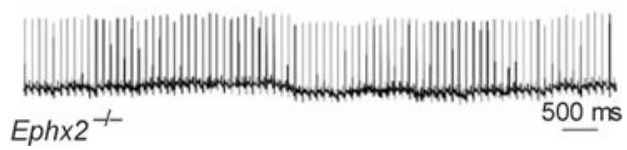

C
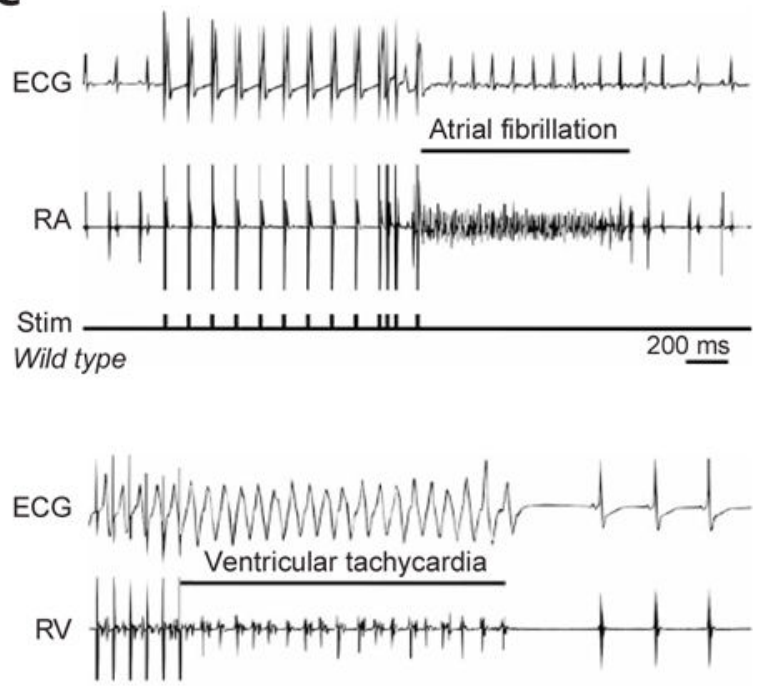

Burst
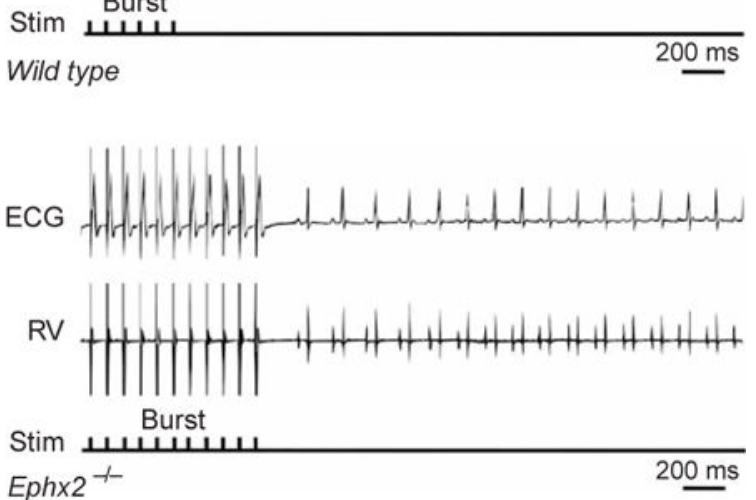

Figure 7.

Electrophysiological characterization of $E p h \times 2^{-/-}$and wild-type mice after Ang II treatment ( $n^{1 / 4} 4$ per group). (a,b) Marked prolongation of ventricular refractoriness (a) and spontaneous premature ventricular contractions $\left(\mathrm{b},{ }^{*}\right)$ were seen in all wild-type mice but could not be observed in any Ephx 2 null mice (values given as mean (central line) \pm s.d.; wide bar, minimum and maximum values). Recordings from a single representative mousefromeach group are given. The lower trace for ECG recordings in a wild-type mouse represents a magnification of the boxedarea from the upper trace. (c) Representative recordings from in vivo electrophysiological studies. Programmed stimulation (stim) in the right ventricle of wild-type mice induced non-sustained atrial fibrillation, indicated by the high frequency signals in the right atrium (RA) and the variability in R-R intervals in the surface ECG (upper panel). Stimulation also induced ventricular tachycardia in wild-type mice. High rate pacing in the right ventricle (RV) is followed by non-sustained fast ventricular tachycardia, eventually recovering into normal sinus rhythm (middle panel). Programmed stimulations could not induce arrhythmias in Ephx $2^{-/-}$mice (lower panel). 


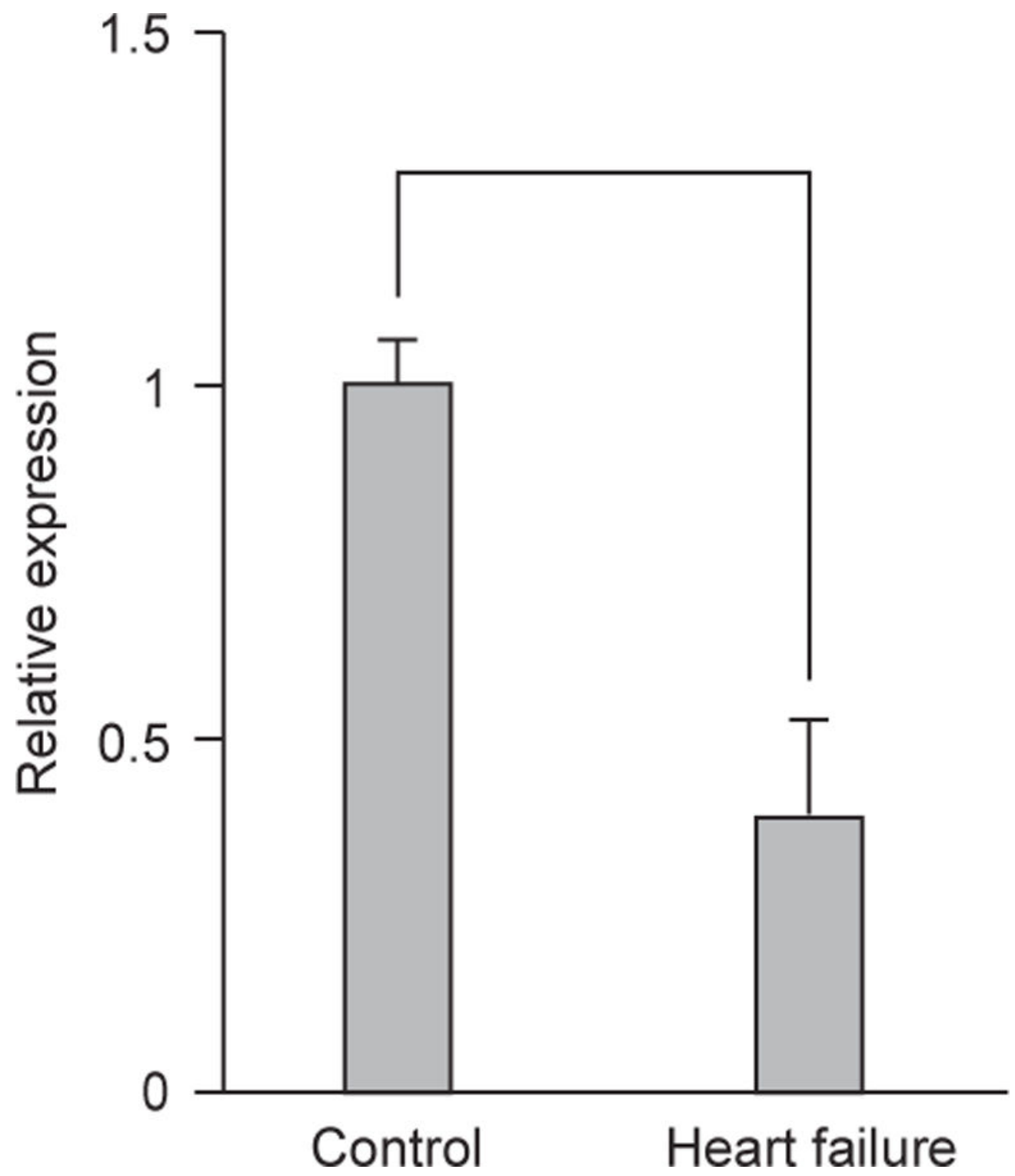

Figure 8.

$E P H X 2$ mRNA expression is downregulated in individuals with heart failure. Ejection fraction in individuals with heart failure was severely reduced ( $n 1 / 46$ per group; ejection fraction $29 \% \pm 5 \%$ in affected individuals versus $450 \%$ in controls), although systolic and diastolic blood pressures were not different between groups. EPHX2 mRNA expression was determined using quantitative TaqMan RT-PCR (values given as mean \pm s.d.; $n^{1 / 4} 6$ per group); * $P^{1 / 4} 0.025$. 